\title{
Spin dynamics and ordering of a cuprate stripe antiferromagnet
}

\author{
G. B. Teitel'baum, ${ }^{*}$ I. M. Abu-Shiekah, O. Bakharev, and H. B. Brom \\ Kamerlingh Onnes Laboratory, Leiden University, P.O. Box 9504, 2300 RA Leiden, The Netherlands \\ J. Zaanen \\ Instituut Lorentz, for Theoretical Physics, Leiden University, P.O. Box 9506, 2300 RA Leiden, The Netherlands
}

(Received 16 October 2000; published 20 December 2000)

\begin{abstract}
In $\mathrm{La}_{1.48} \mathrm{Nd}_{0.4} \mathrm{Sr}_{0.12} \mathrm{CuO}_{4}$ the ${ }^{139} \mathrm{La}$ and ${ }^{63} \mathrm{Cu}$ nuclear quadrupole resonance relaxation rates and signal wipeout upon lowering temperature are shown to be due to purely magnetic fluctuations. They follow the same renormalized classical behavior as seen in neutron data, when the electronic spins order in stripes, with a small spread in spin stiffness (15\% spread in activation energy). The La signal, which reappears at low temperatures, is magnetically broadened and experiences additional wipe out due to slowing down of the $\mathrm{Nd}$ fluctuations.
\end{abstract}

DOI: 10.1103/PhysRevB.63.020507

PACS number(s): 74.72.Dn, 76.60.-k, 75.30.Ds, 75.40.Gb

Strongly correlated electron systems such as layered cuprates exhibit very unusual properties. One of the most interesting among them is the coexistence of superconductivity with local antiferromagnetism (AF) - a fingerprint of the topological effects of doping of AF insulators by holes. The charges segregate into a periodical array of stripes separating antiphase antiferromagnetic domains. Experimental evidence for stripe correlations has been provided by neutron studies in $\mathrm{Nd}$-doped $\mathrm{La}_{1.875} \mathrm{Sr}_{0.125} \mathrm{CuO}_{4}$ and in other cuprates and nickelates. ${ }^{1,2}$ The spatial organization of the stripe structures is a subject of much debate. ${ }^{3-8}$ Stripe formation is characterized by the temperatures of charge ( $\left.T_{\text {charge }}\right)$ and spin $T_{\text {spin }}$ ordering with $T_{\text {charge }}>T_{\text {spin }}$. Since these different types of order coexist on the microscopic level, local methods of analysis, like NMR or nuclear quadrupole resonance (NQR), are well suited to see their interrelation. One striking feature in the NMR data is the wipe-out effect. In $\mathrm{Cu}-\mathrm{NQR}$ experiments on a number of $\mathrm{Sr}$ doped $\mathrm{La}_{2} \mathrm{CuO}_{4}$ samples, Hunt et $a l^{6}$ showed a correlation between the amount of the intensity loss and the development of charge order of the stripe phase. Curro et al. ${ }^{7}$ found strong $\mathrm{Cu}$ wipe-out effect in their NMR experiments on $\mathrm{La}_{2-y-x} \mathrm{Eu}_{y} \mathrm{Sr}_{x} \mathrm{CuO}_{4}$ and showed that this effect could be accounted for by a wide (100\%) distribution in the energy of the thermally activated correlation times that determine the relaxation processes-so-called glassy behavior.

In this communication the role of slow magnetic fluctuations is elucidated. We show that the variation of the line shape as well as wipe-out and relaxation effects probe the growing spin order in the stripe phase. Our investigation takes profit of the NQR frequency range of ${ }^{139} \mathrm{La}$ and ${ }^{63} \mathrm{Cu}$, and especially of the low frequencies and relatively small line widths of $\mathrm{La} \mathrm{NQR}$ in $\mathrm{La}_{1.48} \mathrm{Nd}_{0.4} \mathrm{Sr}_{0.12} \mathrm{CuO}_{4}$. In this compound both $\mathrm{Cu}$ and $\mathrm{La}$ exhibit strong wipe-out effects. Because $\mathrm{La}$ (contrary to $\mathrm{Cu}$ ) nuclei are relatively weakly coupled to the electronic spins in the $\mathrm{CuO}_{2}$ planes, La NQR signals can be followed down to the spin-ordering temperature, as seen by $\mu \mathrm{SR}$. Using the spin correlation times extracted from the activated La spin-lattice relaxation rates we are able to predict precisely these wipe-out features by introducing a spread of only $15 \%$ in the activation energy. Within experimental error this energy agrees with the value found from neutron data, where the relation with stripe ordering was well established, ${ }^{2}$ and is explained in the renormalized classical model. An additional finding is the reappearance of a magnetically broadened NQR signal at low temperatures. For the $6 \mathrm{MHz} \mathrm{La}$ transition the signal intensity is maximal around $4 \mathrm{~K}$, where still about half of the La nuclei are missing.

Experimentally we measure the $T$ dependence of the signal intensities $\widetilde{I}$ and of the relaxation rates of the three ${ }^{139} \mathrm{La}$ NQR transitions $(I=7 / 2)$ at 6,12 , and $18 \mathrm{MHz}$ for $\mathrm{La}_{1.48} \mathrm{Nd}_{0.4} \mathrm{Sr}_{0.12} \mathrm{CuO}_{4}$ and those of ${ }^{63,65} \mathrm{Cu}(I=3 / 2)$ around $36 \mathrm{MHz}{ }^{9}$ The question whether spin or charge fluctuations are relevant is answered by comparison of the rates of the ${ }^{63} \mathrm{Cu}$ and ${ }^{65} \mathrm{Cu}$ and precisely monitoring the magnetization recovery curves after spin reversal for the various La transitions. All relaxation rates are purely due to spin fluctuations. Knowing that the fluctuations are magnetic, we extend the approach of the Los Alamos group ${ }^{7,8}$ to obtain the proper analytic description of the wipe-out effect. With a simple signal visibility criterium and the known values of the hyperfine couplings, from the wipe-out curves correlation times for the spin dynamics are calculated. At the end we show that the La linewidth increase below $20 \mathrm{~K}$ is due to the internal hyperfine field induced by the ordered $\mathrm{Cu}$ moments and that Nd fluctuations are responsible for the missing La NQR signal intensity at the lowest temperatures.

Let us now discuss our findings in more detail. NQR measurements were performed on a powder sample. ${ }^{10}$ The preparation is described in Ref. 11. Susceptibility $\chi$ measurements at $0.001 \mathrm{~T}$ show a superconducting transition temperature of $5 \mathrm{~K}$. The intensity $\widetilde{I}$ multiplied by $T$ and corrected for $T_{2}$ is shown in Fig. 1. Because the nuclear magnetization follows a Curie law, $T \tilde{I}$ is expected to be $T$ independent. This relation is not obeyed, see Fig. 1. Instead, $\widetilde{I} T$ strongly decreases with decreasing $T$, the so-called wipe out being different for $\mathrm{Cu}$ and La. In Fig. 1 arrows indicate the charge $\left(T_{c} \sim 65 \mathrm{~K}\right)$ and spin-order $\left(T_{s} \sim 54 \mathrm{~K}\right)$ temperatures as seen by neutrons, ${ }^{1}$ and the magnetic transition seen by $\mu \mathrm{SR}\left(T_{m} \sim 31 \mathrm{~K}\right){ }^{5}$ The low-temperature orthorhombic (LTO) to lowtemperature tetragonal (LTT) transition is around $68 \mathrm{~K}$.

The spin-lattice $\left(T_{1}^{-1}\right)$ and spin-spin $\left(T_{2}^{-1}\right)$ relaxation rates for the several La-quadrupolar transitions, Fig. 2, peak 


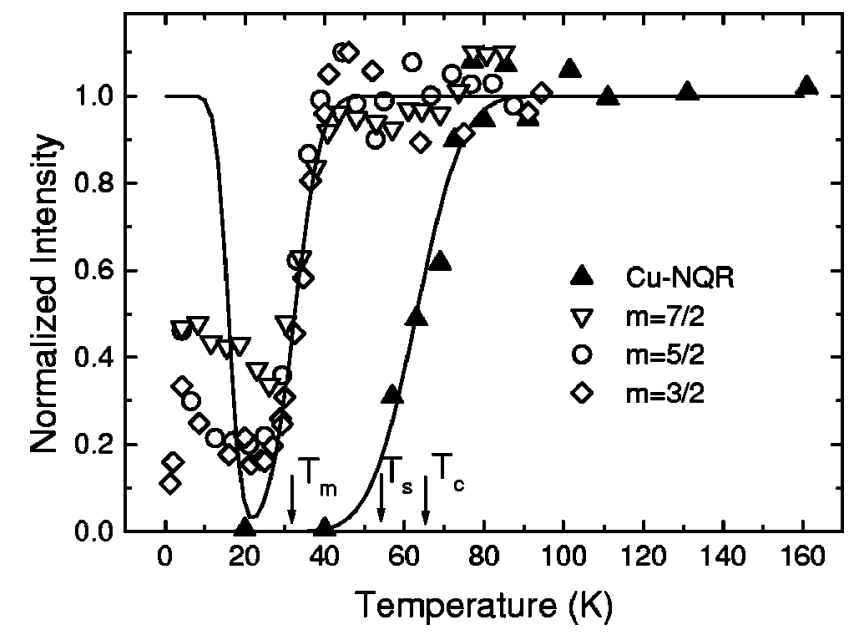

FIG. 1. Wipe out in $\mathrm{La}$ and $\mathrm{Cu} \mathrm{NQR}$. For both $\mathrm{Cu}$ isotopes wipe out starts around $70 \mathrm{~K}$, while for the three satellites of ${ }^{139} \mathrm{La}$, this temperature is around $40 \mathrm{~K}$. Drawn lines are predictions from the model discussed in the text.

around $20 \mathrm{~K}$, where also the wipe out has its maximum. The labels $m=7 / 2,5 / 2$, and $3 / 2$ refer, respectively, to the $( \pm 7 / 2, \pm 5 / 2),( \pm 5 / 2, \pm 3 / 2), \quad$ and $( \pm 3 / 2, \pm 1 / 2)$ transitions. ${ }^{12}$ Fits are made with stretched exponentials ([1$M(t)]$-recovery is $\left.\propto \exp -\left(t / T_{1}\right)^{\alpha}\right)$, indicating the presence of a distribution in rates; the more $\alpha$ deviates from 1 the larger the influence of the distribution is. Here $\alpha$ decreases almost linearly from 1 at $300 \mathrm{~K}$ to 0.6 at $20 \mathrm{~K}$. Down to $30 \mathrm{~K}$ the $T$ dependence can be described by $T_{1}^{-1}=W^{2} \tau /\left(1+\omega^{2} \tau^{2}\right)$ (characteristic for exponential time correlation between fluctuating electronic spins) with $\tau=\tau_{\infty} \exp \left(E / k_{B} T\right),{ }^{13} W$ a matrix element and $E$ an activation energy, see drawn line in Fig. 2. The $T$ dependence of $T_{2}$, see Fig. 2, is determined by the same activation law. From the fit we obtain $E=143 \pm 5 \mathrm{~K}$. With the known hyperfine coupling, ${ }^{14}$ we estimate $\tau_{\infty}$ as $4 \cdot 10^{-12} \mathrm{~s}$. The same value is found from the maximum in $T_{1}^{-1}$.

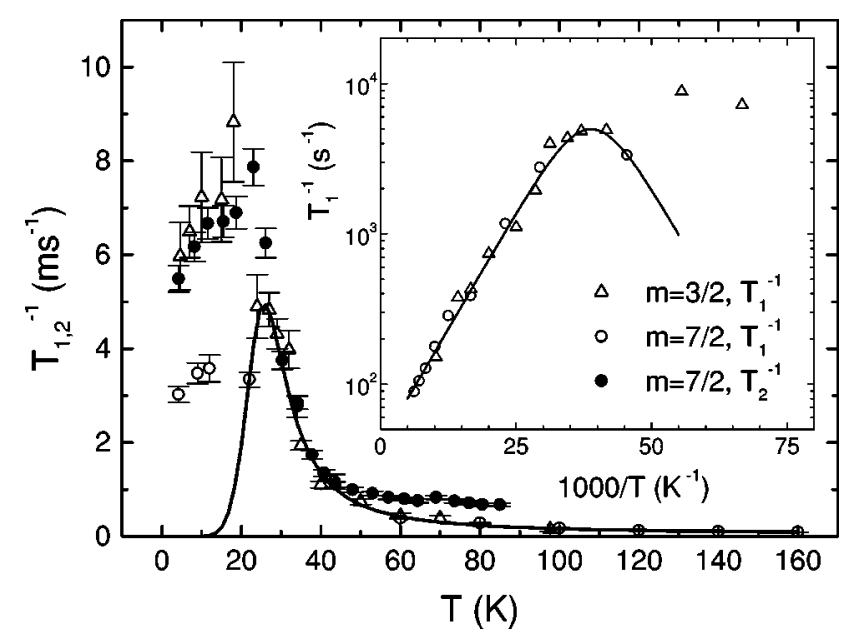

FIG. 2. ${ }^{139} \mathrm{La} T_{1}^{-1}$ and $T_{2}^{-1}$ as function of $T$. The solid line is a fit based on activated behavior with $E_{0}=143 \mathrm{~K}$. The deviations below $20 \mathrm{~K}$ are due to the magnetic ordering.
To see whether the relaxation processes are determined by magnetic or electric fluctuations, we compared the rates for ${ }^{63} \mathrm{Cu}$ and ${ }^{65} \mathrm{Cu}$. The $\mathrm{Cu}$ rates at $71 \mathrm{~K}$ were $8.1\left({ }^{63} \mathrm{Cu}\right)$ and $9.8 \mathrm{~ms}^{-1}\left({ }^{65} \mathrm{Cu}\right)$ and at $130 \mathrm{~K}$, respectively, 7.3 and $10.1 \mathrm{~ms}^{-1}$. If $\omega \tau \ll 1, T_{1}^{-1}$ is proportional to $W^{2} \tau$. For the magnetic case the ratio of the ${ }^{63} \mathrm{Cu}$ and ${ }^{65} \mathrm{Cu}$ transition rates is proportional to $\left(\gamma_{63} / \gamma_{65}\right)^{2}=0.87$, while in case of electric transitions it is the ratio between the quadrupolar moments squared, which equals 1.14 . The found ratio's show $\mathrm{Cu}$ relaxation to be magnetic. For La only the rates for the various quadrupolar transitions are available. Here we make use of the fact that the fundamental transition probability, that appears in the exponents of the relaxation expression is weighted by well defined factors, ${ }^{15}$ that are different for magnetic or electric processes. At 130, 60, 33, 28, and $4.2 \mathrm{~K}$, the magnetization recovery curves after application of a $\pi$ pulse follow stretched exponentials with rates that were a factor $1.8 \pm 0.15$ faster for $m=5 / 2$ than for $m=7 / 2$. This value agrees with the magnetic ratio of $1.9 .^{16}$

How to explain the pronounced wipe-out features? Hunt et al. suggested that the intensity loss might be directly or indirectly related to the growth of the stripe order parameter with decreasing temperature. ${ }^{17}$ Let us restrict ourselves to the case of direct relaxation and to simplify the argument, suppose that the fluctuating stripe order leads to random jumps between the two NQR frequencies which correspond to the extremal values of charge distribution and differ by a value $\delta \omega$. The signal decay for $\delta \omega \tau_{\text {ch }} \ll 1$ can be obtained in a motional narrowing approach ${ }^{13}$ and is given by the $\exp \left\{-t\left[1 / T_{2}+\left(\delta \omega \tau_{\mathrm{ch}}\right)^{2} /\left(8 \tau_{\mathrm{ch}}\right)\right]\right\}$. The resulting decay is not only determined by the dephasing due to magnetic $\left(1 / T_{2}\right)$, but also due to electric fluctuations (lifetime $\tau_{\mathrm{ch}}$ ). Since the experimental relaxation rates turn out to be governed by magnetic fluctuations, charge fluctuations can at most weakly contribute to the wipe-out phenomena. ${ }^{18}$

More generally, wipe-out effects have been shown to be linked to charge/spin fluctuations having a distribution $P(E)$ in activation energies $E$ and hence in correlation times. ${ }^{19,7}$ In case of a Gaussian distribution of $E$, the normalized intensity $I(t)$ is given by

$$
\begin{aligned}
\tilde{I}(t)= & (1 / \sqrt{2 \pi} \Delta) \int_{0}^{\infty} \exp \left[-\left(E-E_{0}\right)^{2} / 2 \Delta^{2}\right] \\
& \times \exp \left[-t / T_{2}(E)\right] d E
\end{aligned}
$$

with $E_{0}$ the mean activation energy, and $\Delta$ the width of the distribution and $\widetilde{I}(0)=1$. In the echo pulse sequences $\pi / 2-t_{r}-\pi-t_{r}$, the delay time $2 t_{r}$ allows a registration in the echo of only those nuclei, that do not relax too fast. Let us assume that we are only seeing those nuclei of which the signal has decayed by a factor of $f$ or less at time $2 t_{r}$, i.e., for which $1 / T_{2 R}=\beta / T_{1}=\left(\Omega^{2} \tau\right) /\left(1+\omega^{2} \tau^{2}\right) \leqslant A .{ }^{20}$ For magnetic fluctuating fields $\Omega^{2}=\beta \gamma^{2} h_{0}^{2}, A=(\ln f) / 2 t_{r}, h_{0}$ denotes the hyperfine field probed by the nuclei and $\beta=(2+r) / 3,{ }^{21}$ with the anisotropy factor $r=3.6$ for $\mathrm{Cu}$ and $\beta=6$ for $\mathrm{La}$ (as deduced from our own relaxation data). The above mentioned inequality determined the boundary values of $\tau$, which follow from $A \omega^{2} \tau^{2}-\Omega^{2} \tau+A=0$ and are given by 
$\tau_{1,2}=\left(\Omega^{2} \pm \sqrt{\Omega^{4}-4 A^{2} \omega^{2}}\right) / 2 A \omega^{2}$. This introduces cut-off's $E_{i}=k_{B} T \ln \left(\tau_{i} / \tau_{\infty}\right)$ in the expression for $\widetilde{I}(t)$, reflecting that part of the nuclei do not contribute to the signal. As a result the extrapolation of $\widetilde{I}\left(2 t_{r}\right)$ to $t=0$ gives

$$
\widetilde{I}_{2 t_{r}}(0) \propto \int_{0}^{E_{2}} e^{-\left(E-E_{0}\right)^{2} / 2 \Delta^{2}} d E+\int_{E_{1}}^{\infty} e^{-\left(E-E_{0}\right)^{2} / 2 \Delta^{2}} d E .
$$

$\widetilde{I}_{2 t_{r}}(0)$ is proportional to the number of nuclei influenced by the magnetic fluctuations with lifetimes outside the interval between $\tau_{1}$ and $\tau_{2}$. There appear two bands in the solution, which contribute to the signal: a band of high-frequency fluctuations (smaller activation energies $E<E_{2}$ ) and a band of low-frequency fluctuations (larger activation energies $E$ $>E_{1}$ ). The values of $E_{1}$ and $E_{2}$ are linear functions of $T$ and the gap $E_{1}-E_{2}=k_{B} T \ln \left(\tau_{1} / \tau_{2}\right)$ between them is the NMR wipe-out gap. The condition for the gap to exist is very simple $\Omega^{2}>2 A \omega$. The presence of two bands, see Eq. (1), gives rise to the reentrant behavior of the echo-amplitude with lowering $T$.

In case of $\mathrm{La} \mathrm{NQR}, \Omega_{\mathrm{La}}$ is rather small, and the condition for the wipe-out gap is realized for $\tau$ lying in the narrow interval around $\tau=\Omega^{2} / 2 A \omega^{2}$. Using $A_{\mathrm{La}} \sim 10^{5} \mathrm{~s}^{-1}\left(f \sim e^{5}\right.$ and $\left.t_{r}=30 \mu \mathrm{s}\right)$, the ${ }^{139} \mathrm{La}$ hyperfine coupling constant $\left(1.7 \mathrm{kOe} / \mu_{\mathrm{B}}\right)^{14}$ and $\Omega_{\mathrm{La}} \sim 6 \cdot 10^{6} \mathrm{~s}^{-1}$ we obtain that for this interval the typical fluctuation times are $\tau \sim 10^{-8} \mathrm{~s}$. For the $\mathrm{Cu}$ nuclei $\Omega^{2} / A \omega^{2} \leqslant \tau \leqslant A / \Omega^{2}$. With $A_{\mathrm{Cu}} \sim A_{\mathrm{La}} \sim 10^{5} \mathrm{~s}^{-1}$, the ${ }^{63} \mathrm{Cu}$ hyperfine coupling constant of $139 \mathrm{kOe} / \mu_{\mathrm{B}}$, and $\Omega_{\mathrm{Cu}} \sim 6 \cdot 10^{8} \mathrm{~s}^{-1}$, it follows that the wipe-out at $75 \mathrm{~K}$ is due to the fluctuations with $\tau \sim 10^{-11}-10^{-12}$ s. Neglecting effects of the magnetic ordering of $\mathrm{Cu}$ (and the Nd) moments, the reappearance of the $\mathrm{Cu}$ NQR signal will take place for extremely slow fluctuations with $\tau \sim 10^{-6} \mathrm{~s}$, realized only at very low temperatures.

The drawn lines in Fig. 1 are fits to the wipe-out behavior with the numerical constants calculated above. The free parameters are in principle $E_{0}, \Delta$, and $\ln \left(\tau_{i} / \tau_{\infty}\right)$, with $\tau_{1,2}$ being fixed by $A$ and $\Omega$. If for $E_{0}$ the same value is used as for the relaxation data, i.e., $E_{0}=143 \pm 5 \mathrm{~K}$, the fit to the $\mathrm{Cu}$ and La data gives mutually consistent values for the other free parameters: $\Delta=21 \pm 3 \mathrm{~K}$ and $\tau_{\infty}$ equals the value found from the relaxation data. Note that for the low frequency La transitions the wipe-out is more pronounced, since the wipeout gap is $\propto 1 / \omega^{2}$.

An activated $T$ dependence of $\tau$ can have many causes. However, a most natural interpretation is in terms of the behavior of the relaxation time of a classical quasi-twodimensional (2D) Heisenberg antiferromagnet which is on its way to its 3D phase transition. The relaxation time is set by the magnetic correlation length $\xi,{ }^{22}$ and the latter behaves like $\xi(T)=e^{T * / T} /\left(2 T^{*}+T\right)$ where $T^{*}=2 \pi \rho_{s}$ in terms of the spin stiffness $\rho_{s}$. According to our relaxation and wipeout data $T^{*}=143 \pm 5 \mathrm{~K}$ which is consistent with the $T^{*}$ $=200 \pm 50 \mathrm{~K}$ as deduced by Tranquada et al. ${ }^{2}$ from the $T$ dependence of $\xi$ as measured by neutron scattering. This spin stiffness associated with the stripe antiferromagnet is an order of magnitude smaller than the one of the pure antifer-

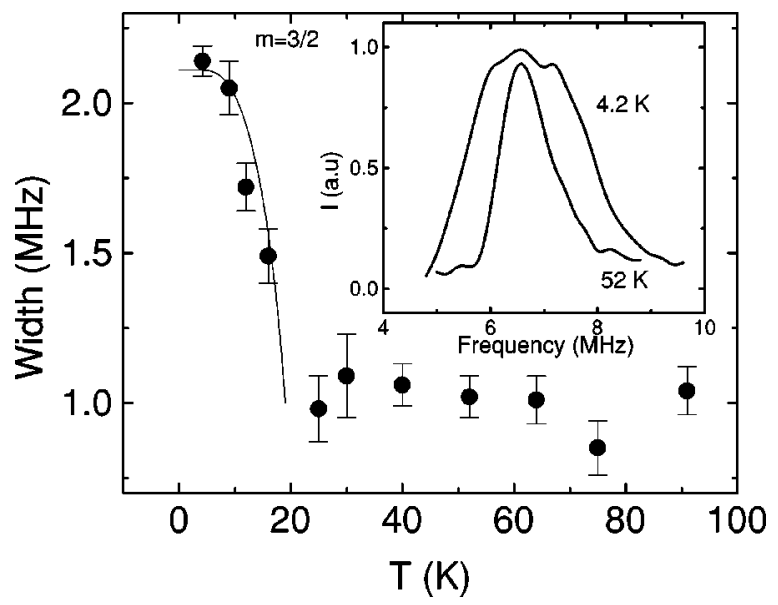

FIG. 3. $T$ dependence of the ${ }^{139} \mathrm{La}$ linewidth for $m=3 / 2$. Magnetic ordering sets in below $20 \mathrm{~K}$ (drawn line: mean-field fit). The inset shows the changes in the line profile.

romagnet of half filling. If the spin system would be classical the implication would be that the exchange interactions mediated by the charge stripes would be smaller by two orders of magnitude as compared to the exchange interaction inside the magnetic domains. This is inconsistent with the persistence of antiphase correlations up to rather high energies as seen by inelastic neutrons scattering. Moreover, there is no doubt that the spin system is highly quantum-mechanical at short length scales and the stripe antiferromagnet should exhibit renormalized classical behavior. ${ }^{23}$ This implies that the spin system should be in the proximity of a quantum-phase transition to a disordered state and it is well understood that the renormalized stiffness diminishes when this transition is approached, while the spin velocity is barely changing. Hence, the small spin-stiffness of the stripe antiferromagnet signals that this system is much closer to the quantum phase transition than the half-filled antiferromagnet, in agreement with theoretical expectations. ${ }^{24,25}$

To evaluate the role of the $\mathrm{Nd}$ ion on the correlation times, we also determined the relaxation rates in $\mathrm{La}_{1.71} \mathrm{Eu}_{0.17} \mathrm{Sr}_{0.12} \mathrm{CuO}_{4} \cdot{ }^{6,8,26}$ The ${ }^{139} \mathrm{La}$ relaxation rates were about a factor 10 lower than in the $0.4 \mathrm{Nd}$ compound. With the hyperfine coefficients used in $0.4 \mathrm{Nd}$, the correlation times found for the fluctuating fields derived from $T_{1}^{-1}\left({ }^{63} \mathrm{Cu}\right)$ and $T_{1}^{-1}\left({ }^{139} \mathrm{La}\right)$ above $20 \mathrm{~K}$ in the Eu sample were the same, but compared to the Nd sample, the values of $\tau$ at comparable temperatures are different. It likely reflects the different pinning strength of stripe structure with the inhomogeneities of LTT phase induced by the $\mathrm{Nd}$ and Eu ion, whereas the equal hyperfine constants show that above $20 \mathrm{~K}$ Nd does not influence the La nuclear relaxation rates directly.

To determine whether spins or charges are responsible for the final La line shapes, we have followed the line profiles for various satellites (due to its small splitting, $m=3 / 2$ is the most sensitive) as function of $T$, see Fig. 3. Above the wipeout regime the La line widths scale with their splitting, which show them to be electric. Below $20 \mathrm{~K}$ the linewidths increase due to the presence of an internal magnetic field, see Fig. 3. The drawn line represents the mean-field staggered magneti- 
zation for $S=1 / 2$. The saturated value of the additional width (full width at half intensity), obtained by taking the square root of the difference in second moments of the broadened and unbroadened line, amounts to $2.0 \mathrm{MHz}$, close to the splitting seen in undoped $\mathrm{La}_{2} \mathrm{CuO}_{4}$, where the $m$ $=3 / 2$ splitting is $2.5 \mathrm{MHz}^{27}$ In the undoped compound (with an ordered moment in the Neel state of $\sim 0.55 \mu_{B}$ ) the splitting can be reproduced by a field of $0.11 \mathrm{~T}$ perpendicular to the electric field gradient (with anisotropy parameter $\eta$ $=0.02$ and in plane field angle $\phi=0)$. Here the saturated splitting seen for the $m=3 / 2$ line can be simulated by an external field of $0.08 \mathrm{~T}$, again applied perpendicular to the electric field gradient ( $\eta=0.13$ is fixed by the line positions above the magnetic ordering and $\phi=\pi / 4$ ). As (see below) $\mathrm{Nd}$ moments are not yet involved, we estimate the $\mathrm{Cu}$ ordered moment in $0.4 \mathrm{Nd}$ to be $\sim 0.4 \mu_{B}$. The missing spectral weight of about $50 \%$ at $4.2 \mathrm{~K}$ for $m=3 / 2(\mathrm{La})$ might be explained by an internal field of the same order as the quadrupolar splitting of $6 \mathrm{MHz}$ felt by the unseen La sites. Such a scenario agrees with the $\mu \mathrm{SR}$ finding ${ }^{5}$ that most or all $\mu \mathrm{SR}$ sites are magnetic. However, $0.1 \mathrm{~T}(2 \mathrm{MHz})$ at $4.2 \mathrm{~K}$ is about the maximum field at the $\mathrm{La}$ sites (even with $\mathrm{Nd}^{2,4,10}$ ) one might expect. The $T$ dependence of $\widetilde{I}(m=3 / 2)$ below $4.2 \mathrm{~K}$ shows that we deal with additional wipe out caused by slow
Nd-spin fluctuations. This extra channel in $T_{2}^{-1}(\mathrm{La})$ becomes important close to the ordering temperature of $1 \mathrm{~K}$ of the $\mathrm{Nd}$ moments ${ }^{10}$ and partially destroys the recovery of echo-signal predicted by Eq. (1).

In summary, the wipe-out and relaxation features of $\mathrm{Cu}$ and $\mathrm{La}$ in the temperature regime above the spin-ordering transition find a natural explanation in terms of the well understood fluctuations of a quantum antiferromagnet which is approaching its ordered state. For the $0.4 \mathrm{Nd}$ compound this "ordered" state is not straightforward as wipe out persists down to $1 \mathrm{~K}$ for the majority of La spins. Here La wipe out proceeds in two stages, of which the first is due to slowing down of $\mathrm{Cu}$ spins and the second below $4 \mathrm{~K}$ is dominated by fluctuations of Nd magnetic moments. Form the La NQR lineshape we estimate an ordered $\mathrm{Cu}$ moment of $0.4 \mu_{B}$ in the stripe phase.

This work was supported in part by the Dutch Science Foundation FOM-NWO and by the State HTSC Program of the Russian Ministry of Sciences (Grant No. 98001) and by the Russian Foundation for Basic Research (Grant No. 9802-16528). O.G.A. Berfelo is acknowledged for his assistance in the measurements.
*Permanent address: Institute of Technical Physics of the Academy of Sciences of Russia, 420029 Kazan, Russia.

${ }^{1}$ J. M. Tranquada, B. J. Sternlieb, J. D. Axe, Y. Nakamura, and S. Uchida, Nature (London) 375, 561 (1995).

${ }^{2}$ J. M. Tranquada, N. Ichikawa, and S. Uchida, Phys. Rev. B 59, 14712 (1999).

${ }^{3}$ J. Zaanen, Nature (London) 404, 714 (2000); Science 286, 251 (1999); V. J. Emery, S. A. Kivelson, and J. M. Tranquada, Proc. Natl. Acad. Sci. U.S.A. 96, 8814 (1999), and references therein.

${ }^{4}$ M. Roepke et al., Phys. Rev. B 60, 9793 (1999).

${ }^{5}$ B. Nachumi et al., Phys. Rev. B 58, 8760 (1998).

${ }^{6}$ A. W. Hunt, P. M. Singer, K. R. Thurber, and T. Imai, Phys. Rev. Lett. 82, 4300 (1999); P. M. Singer, A. W. Hunt, A. F. Cederström, and T. Imai, Phys. Rev. B 60, 15345 (1999).

${ }^{7}$ N. J. Curro et al., Phys. Rev. Lett. 85, 642 (2000).

${ }^{8}$ B. J. Suh et al., Phys. Rev. B 61, 9265 (2000).

${ }^{9}$ For $\widetilde{I}(\mathrm{La})$ the whole line is integrated and if needed corrected for sensitivity changes with $\omega . \widetilde{I}(\mathrm{Cu})$ refers to peak values of the line and is therefore sensitive for changes in lineshape, which occur below $20 \mathrm{~K}$.

${ }^{10}$ G. B. Teitel'baum, E. L. Vavilova, B. Büchner, and H. Luetgemeier, Pis'ma Zh. Éksp. Teor. Fiz. 67, 344 (1998) [JETP Lett. 67, 363 (1998)].

${ }^{11}$ B. Büchner et al., Physica C 185-189, 903 (1991); Europhys. Lett. 21, 953 (1993).

${ }^{12}$ In case of magnetic order the $\pm 1 / 2$ state will no longer be pure (Ref. 27).

${ }^{13}$ C. P. Slichter, Principles of Magnetic Resonance, 3rd ed.
(Springer-Verlag, New York, 1990), pp. 197 and 595.

${ }^{14}$ T. Goto, S. Kazama, K. Miyagawa, and T. Fukase, J. Phys. Soc. Jpn. 63, 3494 (1994).

${ }^{15}$ I. Watanabe, J. Phys. Soc. Jpn. 63, 1560 (1994); J. Chepin and J. H. Ross, Jr., J. Phys.: Condens. Matter 3, 8103 (1991).

${ }^{16}$ The factor 1.9 results from a fit of the theoretical magnetic recovery curves for NQR lines to a stretched exponential.

${ }^{17}$ Charge ordering in stripes will not only slow down charge fluctuations but might also change the magnetic fluctuation spectrum considerably, inducing wipe out (see Ref. 6).

${ }^{18}$ The decay by $\tau_{\text {ch }}$ is the strongest for $\delta \omega \tau_{\text {ch }} \sim 1$, when the echo signal is already suppressed by magnetic fluctuations.

${ }^{19}$ The activated behavior is suggested by the relaxation data and biased by theory (see last part).

${ }^{20}$ R. L. Corey et al., Phys. Rev. B 53, 5907 (1996).

${ }^{21}$ R. Stern, M. Mali, J. Roos, and D. Brinkmann, Phys. Rev. B 51, 15478 (1995).

${ }^{22}$ S. Tyč, B. I. Halperin, and S. Chakravarty, Phys. Rev. Lett. 62, 835 (1989).

${ }^{23}$ S. Chakravarty, B. I. Halperin, and D. R. Nelson, Phys. Rev. B 39, 2344 (1989).

${ }^{24}$ C. N. A. van Duin and J. Zaanen, Phys. Rev. Lett. 80, 1513 (1998); J. Tworzydlo, O. Y. Osman, C. N. A. van Duin, and J. Zaanen, Phys. Rev. B 59, 115 (1999).

${ }^{25}$ S. Sachdev, Science 288, 475 (2000).

${ }^{26}$ G. B. Teitel'baum, B. Büchner, and H. de Gronckel, Phys. Rev. Lett. 84, 2949 (2000).

${ }^{27}$ D. E. MacLaughlin et al., Phys. Rev. Lett. 72, 760 (1994). 